\title{
Swyer-James syndrome with vascular ring in a child: Echocardiogrpahic diagnosis
}

\author{
Fong Lin Chen ${ }^{* 1,2}$, Wei Cheng $\mathrm{Chen}^{3}$, Shu Min Kuo ${ }^{3}$ \\ ${ }^{1}$ Division of Pediatric Cardiology, Department of Pediatrics, Chung-Shan Medical University Hospital, Taichung, Taiwan \\ ${ }^{2}$ Taiwan Institute of Medicine, Chung-Shan Medical University, Taichung, Taiwan \\ ${ }^{3}$ Division of Cardiovascular Surgery, Chung-Shan Medical University Hospital, Taichung, Taiwan
}

Received: January 31, 2016

DOI: $10.5430 /$ jbgc.v6n2p1
Accepted: March 14, $2016 \quad$ Online Published: April 29, 2016

URL: http://dx.doi.org/10.5430/jbgc.v6n2p1

\begin{abstract}
Swyer-James syndrome (SJS) is a relatively uncommon type of postinfectious obliterative bronchiolitis as a result of respiratory tract infection (RTI) in early childhood. Vascular ring (VR) is usually an underlying cause of RTI. When VR complicated with SJS, both diseases can be a relationship of cause and result, but have not been published in the past. Herein, we described an 8 -year-old female school-age child who had history of recurrent RTI since age of 6 months. After screening echocardiographic findings of hypoplastic left pulmonary artery and aberrant right subclavian artery (ARSCA), confirmed by multisliced CT and functional evaluation by bronchoscopy and manometry. After she underwent aortopexy for ARSCA and balloon dilatation of constrictive bronchus and tracheal stenosis, and her growth pattern and pulmonary function improved progressively. Early detection by echocardiography and elimination of the associated underlying airway obstruction were mandatory for improvement of pulmonary function.
\end{abstract}

Key Words: Aberrant right subclavian artery, Bronchiolitis obliterans, Respiratory tract infection, Swyer-James syndrome

\section{INTRODUCTION}

Vascular ring (VR) with aberrant right subclavian artery (ARSCA) had a sling effect on trachea and esophagus to cause variable tracheoesophageal compression (TEC). ARSCA is not only a common cause of dysphagia lusoria to lead to failure to thrive but also as a cause of airway obstruction to result in frequent respiratory tract infection. ${ }^{[1-3]}$ Swyer-James syndrome (SJS), a rare type of post-infectious bronchiolitis obliterans $(\mathrm{BO}){ }^{[4]}$ is usually characterized by unilateral hyperlucency of part of or whole of lung usually following a respiratory infection in infancy or early childhood. ${ }^{[5]}$ Pulmonary hypertension can be a bad outcome of SJS due to progressive pulmonary function impairment. When VR complicated with SJS, the exacerbation of airway obstruc- tion by VR can augment pulmonary function impairment in SJS. Early detection of underlying airway obstruction and effective optimization of airway patency were mandatory to reduce RTI. Unique echocardiographic features of a unilateral hypoplastic pulmonary artery and abnormal aortic branching can make echocardiography an effective tool for early detection of VR and SJS. Herein, we describe an 8year-old girl with both diseases and significant pulmonary function impairment. The echocardiographic diagnosis, treatment and outcome of SJS complicated by VR were firstly emphasized.

\section{CASE REPORT}

This 8-year-old girl was admitted due to small left pulmonary artery and aberrant right subclavian artery incidentally found

*Correspondence: Fong Lin Chen; Email: cshy053@gmail.com; Address: 2F., No.375, Sanmin W. Rd., South Dist., Taichung City 402, Taiwan. 
by screening echocardiography (see Figure 1). She usually suffered from recurrent respiratory tract infection since age of 6 months, dyspnea on exercise with poor tolerance, poor appetite with difficulty in taking meat nugget since childhood. She was usually treated as asthma and got failure to thrive. Due to symptoms of TEC, she was admitted to evaluate the indication for further treatment. Physical examination showed decreased breathing sound without rales or rhonchi on left chest and scoliosis. Chest radiograph showed hyperluscency and small hilum in left lung and hyperinflation of right lung (see Figure 2). Multislice computed tomography (MSCT) showed hypoplasia of left pulmonary artery and ARSCA compatible with echocardiogaphic findings, small left lower lobe and left hemithorax with diffuse decrease in attenuation, paucity of bronchovascular markings without proximal bronchiectasis in left hemithorax and upper tracheal and esophageal compression by external sling of ARSCA (see Figure 3).

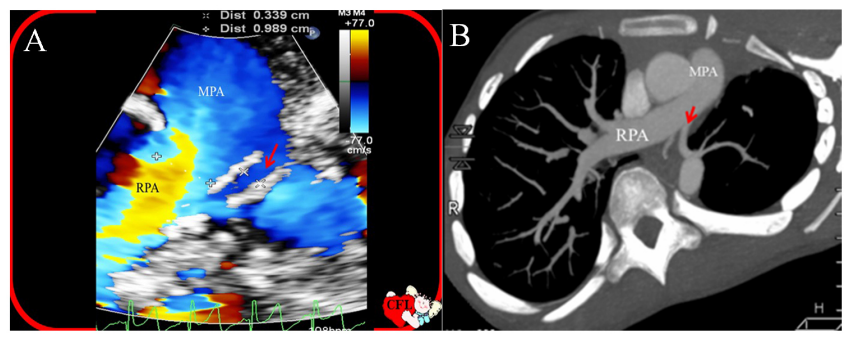

Figure 1. Hypoplastic left pulmonary artery (RPA) was revealed as (A) an unique branching (red arrow) from major pulmonary artery (MPA) on echocardiography and confirmed by (B) MSCT with decreased volume of left lung and bronchovascular markings as typical pruned tree

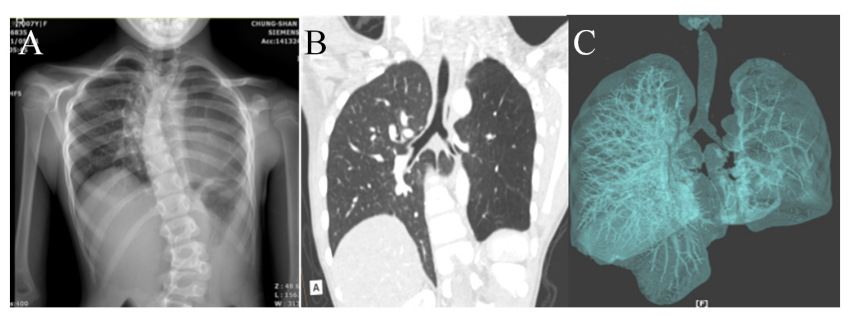

Figure 2. (A) Chest x-ray showed a small, hyperexpanded, hyperlucent, hypovascular left lung. With scoliosis (B) Coronal reformatted CT image shows a hyperlucent, hyperexpanded left lung and distal obstruction of left bronchus (arrow-head). (C) Volume-rendered images of the airway may depict distal narrowing of left bronchus with a small, hyperexpanded, hyperlucent, hypovascular left lung

Hypoplastic left pulmonary artery and typical pruned tree appearance of pulmonary vasculatures of left lung confirmed the diagnosis of SJS. Pulmonary function showed small airway with restrictive ventilatory impairment (FVC $0.62 \mathrm{~L}$ [45\% of predicted value], FEV1 $0.45 \mathrm{~L}$ [36\% of predicted value]). Mild pulmonary hypertension with dilated right ventricle and pulmonary artery pressure of $30 \mathrm{mmHg}$ by estimation of tricuspid valvular regurgitation was found on echocardiography. Due to significant tracheal and bronchial narrowing on bronchoscopy and a localized compression zone with high pressure of $103 \mathrm{mmHg}$ on manometry, she underwent aortopexy and bronchial dilation to optimize airway patency. She had complete relief of TEC by VR and progressive improvement of growth pattern and pulmonary function.

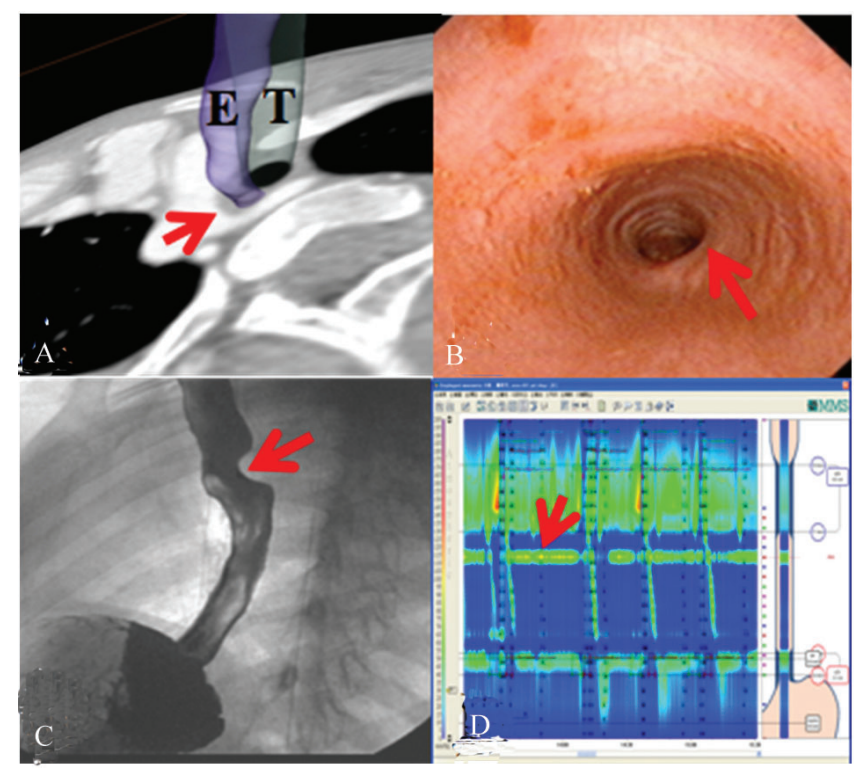

Figure 3. Axial reformatted CT image showed (A) a symptomatic vascular ring with aberrant right subclavian artery to suppose a sling effect on trachea and esophagus to cause significant tracheal narrowing on bronchoscopy (B) and indentation on esophagogram (C) with a localized high pressure zone of $103 \mathrm{mmHg}$ on manometry (D). Trachea (T), esophagus (E), external compression (red arrow)

\section{Discussion}

Despite recurrent respiratory infection since childhood and characteristic findings by chest radiography, diagnosis of SJS still is a challenge. ${ }^{[6]}$ By the unique hypoplasia and/or agenesis of the ipsilateral pulmonary arteries, echocardiography can play an effective alternative for detection of SJS and associated cardiac lesions. The prevalence of SJS after bronchiolitis obliterans is not known, but is firstly reported as one in 345,000 schools children by screening echocardiography in our study. ${ }^{[7]}$ Our data was less than $0.01 \%$ in 17,450 chest radiographic survey. ${ }^{[8]}$ MSCT was more useful than MRI as 
the golden standard to confirm the diagnosis and evaluate the airway and lung parenchymal impairment. ${ }^{\text {[9] }}$

Prevention and management of recurrent respiratory tract infections is the main treatment for SJS. ${ }^{[10]}$ If infections of the ectatic bronchi cannot be controlled, advanced treatment to remove a diseased and chronically infected lung to allow normal development of lung parenchyma is essential for improvement of quality of life and life expectancy. VR with ARSCA, a sling effect on trachea and esophagus with variable degree of airway obstruction, is usually a cause of respiratory tract infection. By the complications of vascular ring with SJS, there will not only increase the comorbidity of RTI and obstructive pneumonia but also exacerbate the pulmonary function in SJS.

To our knowledge, this case of VR complicated SJS has not been reported. VR and SJS can be viewed as a relationship of cause and result. Airway obstruction can be an underlying and exacerbated cause of bronchiectasis in SJS. Although isolate ARSCA was considered to be asymptomatic and need not be treated, surgery for vascular ring complicating with SJS will be essential for relief of airway obstruction to decrease the risk of recurrent RTI and pulmonary function impairment.

Through the case findings, unique echocardiographic feature of hypoplastic or agenesis of ipsilateral pulmonary artery is useful for us to early detect SJS and associated underlying cause of airway obstruction. Treatment of airway obstruction can effectively reduce recurrent RTI to herald the downward progression of lung function in SJS.

\section{CONFlicts OF INTEREST Disclosure}

Authors of the article do not have financial and personal relationships with other people or organizations that can inappropriately influence our work, there is no professional or other personal interest of any nature or kind in any product, service and/or company that could be construed as influencing the position presented in, or the review of the manuscript entitled.

\section{REFERENCES}

[1] Subramanyan R, Venugopalan P, Narayan R. Vascular Rings: An important cause of persistent respiratory symptoms in infants and children. Indian Pediatr. 2003; 40: 951-957. PMid:14581732.

[2] van Aalderen WM, Hoekstra MO, Hess J, et al. Respiratory infections and vascular rings. Acta Prediatr Scand. 1990; 79: 477-480. http: //dx.doi.org/10.1111/j.1651-2227.1990.tb11500.x

[3] Carrizo GJ, Marjani MA. Dysphagia lusoria Caused by an aberrant right Subclavian artery. Tex Heart Inst J. 2004; 31(2): 168-171. PMid:15212130.

[4] Schlesinger C, Meyer CA, Veeraraghavan S, et al. Constrictive (obliterative) bronchiolitis: diagnosis, etiology, and a critical review of the literature. Ann Diagn Pathol. 1998; 2: 321-334. http: //dx.doi.org/10.1016/S1092-9134(98)80026-9

[5] Scan J Infect Dis. Swyer-James (MacLeod's) syndrome following pertussis infection in an infant. Scand J Infect Dis. 2003; 35(3): 197199. http://dx.doi.org/10.1080/0036554031000027017

[6] Kim CK, Koh JY, Han YS, et al. Swyer-James Syndrome with finger clubbing after severe measles infection. Pediatr Int. 2008; 50(3):
413-415. PMid:18533964. http://dx.doi.org/10.1111/j.144 2-200X. 2008.02597.x

[7] Chen FL, Ge SP. Congenital Vascular Rings And Slings Are A Significantly Under-diagnosed Cause Of Childhood Diseases: Screening Of 186,213 School-aged Children By Echocardiography. Abstract present at: ACC.07 and the i2 Summit of the the American College of Cardiology; March 25, 2007; Louisiana, LA

[8] Gaensler EA. Unilateral hyperlucent lung. In: Simon M, Potchen E, LeMay M, editors. Frontiers of pulmonary radiology. New York: Grune \& Stratton; 1969. p. 312-319.

[9] Gómez Belda AB, Martínez-Moragón E, Fernández Fabrellas E. Swyer-James syndrome: diagnostic contributions of helical computerized tomography. Arch Bronconeumol. 2000; 36: 421. http: //dx.doi.org/10.1016/S0300-2896(15) 30145-9

[10] Fregonese L, Girosi D, Battistini E, et al. Clinical, physiologic, and roentgenographic changes after pneumonectomy in a boy with Macleod/Swyer-James syndrome and bronchiectasis. Pediatr Pulmonol. 2002; 34: 412-6. PMid:12357493. http://dx.doi.org/1 $0.1002 / \mathrm{ppul} .10178$ 